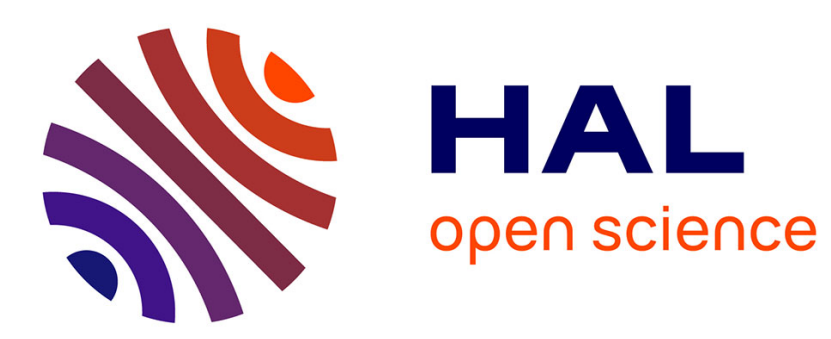

\title{
Verification of EB3 Specifications Using CADP
}

Dimitris Vekris, Frédéric Lang, Catalin Dima, Radu Mateescu

\section{To cite this version:}

Dimitris Vekris, Frédéric Lang, Catalin Dima, Radu Mateescu. Verification of EB3 Specifications Using CADP. iFM 2013 - 10th International Conference on integrated Formal Methods, Jun 2013, Turku, Finland. 10.1007/978-3-642-38613-8_5 . hal-00768310v4

\section{HAL Id: hal-00768310 https://hal.inria.fr/hal-00768310v4}

Submitted on 26 Apr 2013

HAL is a multi-disciplinary open access archive for the deposit and dissemination of scientific research documents, whether they are published or not. The documents may come from teaching and research institutions in France or abroad, or from public or private research centers.
L'archive ouverte pluridisciplinaire HAL, est destinée au dépôt et à la diffusion de documents scientifiques de niveau recherche, publiés ou non, émanant des établissements d'enseignement et de recherche français ou étrangers, des laboratoires publics ou privés. 


\title{
Verification of $\mathrm{EB}^{3}$ Specifications using CADP
}

\author{
Dimitris Vekris ${ }^{1}$, Frédéric Lang ${ }^{2}$, Catalin Dima $^{1}$, Radu Mateescu $^{2}$ \\ 1 LACL, Université Paris-Est \\ 61, av. du Général de Gaulle, F-94010 Créteil, France \\ \{Dimitrios.Vekris, Catalin.Dima\}@u-pec.fr \\ 2 Inria Grenoble Rhône-Alpes and LIG - CONVECS team \\ 655, av. de l'Europe, Montbonnot, F-38334 Saint Ismier, France \\ \{Frederic.Lang, Radu.Mateescu\}@inria.fr
}

\begin{abstract}
EB}^{3}$ is a specification language for information systems. The core of the $\mathrm{EB}^{3}$ language consists of process algebraic specifications describing the behaviour of the entities in a system, and attribute function definitions describing the entity attributes. The verification of $\mathrm{EB}^{3}$ specifications against temporal properties is of great interest to users of $\mathrm{EB}^{3}$. In this paper, we propose a translation from $\mathrm{EB}^{3}$ to LOTOS NT (LNT for short), a value-passing concurrent language with classical process algebra features. Our translation ensures the one-to-one correspondence between states and transitions of the labelled transition systems corresponding to the $\mathrm{EB}^{3}$ and LNT specifications. We automated this translation with the $\mathrm{EB}^{3}$ 2LNT tool, thus equipping the $\mathrm{EB}^{3}$ method with the functional verification features available in the CADP toolbox.
\end{abstract}

\section{Introduction}

The $\mathrm{EB}^{3}$ method [10] is an event-based paradigm tailored for information systems (ISs). $\mathrm{EB}^{3}$ has been used in the research projects SELKIS [19] and $\mathrm{EB}^{3} \mathrm{SEC}$ [17], whose primary aim is the formal specification of ISs with security policies. In the $\mathrm{EB}^{3} \mathrm{SEC}$ project, real banking industry case studies have been studied, describing interaction with brokers, customers and external financial systems. The SELKIS project deals with two case studies from the medical domain. The first one draws data records from medical imaging devices. The access to these records is done via web-based applications. The second one deals with availability and confidentiality issues for medical emergency units evolving in a great mountain range, like the Alps in that case.

A typical $\mathrm{EB}^{3}$ specification defines entities, associations, and their respective attributes. The process algebraic nature of $\mathrm{EB}^{3}$ enables the explicit definition of intra-entity constraints, making them easy for the IS designer to review and understand. Yet, its particular feature compared to classical process algebras,

\footnotetext{
^ Partially supported by SELKIS ANR Project. The LACL group is grateful to CONVECS for its warm welcome to INRIA Grenoble in 2012 and wishes to thank all its members for useful advice and discussions.
} 
such as CSP [15], lies in the use of attribute functions, a special kind of recursive functions evaluated on the system execution trace. Combined with guards, attribute functions facilitate the definition of complex inter-entity constraints involving the history of events. The use of attribute functions simplifies system understanding, enhances code modularity, and streamlines maintenance. However, given that ISs are complex systems involving data management and concurrency, a rigorous design process based on formal specification using $\mathrm{EB}^{3}$ must be completed with effective formal verification features.

Existing attempts for verifying $\mathrm{EB}^{3}$ specifications are based on translations from $\mathrm{EB}^{3}$ to other formal methods equipped with verification capabilities. A first line of work $[13,14]$ focused on devising translations from $\mathrm{EB}^{3}$ attribute functions and processes to the $\mathrm{B}$ method [2], which opened the way for proving invariant properties of $\mathrm{EB}^{3}$ specifications using tools like Atelier B [6]. Another line of work concerned the verification of temporal logic properties of $\mathrm{EB}^{3}$ specifications by means of model checking techniques. For this purpose, the formal description and verification of an IS case-study using six model checkers was undertaken in $[9,5]$. This study revealed the necessity of branching-time logics for accurately characterizing properties of ISs, and also the fact that process algebraic languages are suitable for describing the behaviour and synchronization of IS entities. However, no attempt of providing a systematic translation from $\mathrm{EB}^{3}$ to a target language accepted as input by a model checker was made so far.

In this paper, we aim at filling this gap by proposing a translation from $\mathrm{EB}^{3}$ to LNT [7], a new generation process algebraic specification language inspired from E-LOTOS [16]. As far as we know, this is the first attempt to provide a general translation from $\mathrm{EB}^{3}$ to a classical value-passing process algebra. It is worth noticing that CSP and LNT were already considered in [9] for describing ISs, and identified as candidate target languages for translating $\mathrm{EB}^{3}$. Since our primary objective was to provide temporal property verification features for $\mathrm{EB}^{3}$, we focused our attention on LNT, which is one of the input languages accepted by the CADP verification toolbox [11], and hence is equipped with on-the-fly model checking for action-based, branching-time logics involving data.

At first sight, given that $\mathrm{EB}^{3}$ has structured operational semantics based on a labelled transition system (LTS) model, its translation to a process algebra may seem straightforward. However, this exercise proved to be rather complex, the main difficulty being to translate a history-based language to a process algebra with standard LTS semantics. To overcome this difficulty, we considered alternative memory-based semantics of $\mathrm{EB}^{3}$ [20], which were shown to be equivalent to the original trace-based semantics defined for finite-state systems in [10]. Another important ingredient of the translation was the multiway value-passing rendezvous of LNT, which enabled to obtain a one-to-one correspondence between the transitions of the two LTSs underlying the $\mathrm{EB}^{3}$ and LNT descriptions, and hence to preserve strong bisimulation. The presence of array types and of usual programming language constructs (e.g., loops and conditionals) in LNT was also helpful for specifying the memory, the Kleene star-closure operators, and the $\mathrm{EB}^{3}$ guarded expressions containing attribute function calls. At last, 


$$
\begin{aligned}
E B^{3}:: & =A_{1} ; \ldots ; A_{n} ; S_{1} ; \ldots ; S_{m} \\
A::= & f(\mathrm{~T}: \mathcal{T}, \bar{y}: \bar{T}): T=\text { match last }(\mathrm{T}) \text { with } \\
& \quad \quad\left(\bar{v} v_{0}\left|\alpha_{1}\left(\overline{x_{1}}\right): v_{1}\right| \ldots \mid \alpha_{q}\left(\overline{x_{q}}\right): v_{q}\left[\mid-: v_{q+1}\right]\right. \\
S::= & P(\bar{x})=E \\
E::= & \lambda|\alpha(\bar{v})| E_{1} . E_{2}\left|E_{1}\right| E_{2}\left|E_{0}{ }^{*}\right| E_{1}|[\Delta]| E_{2}|| x: V: E_{0} \mid \\
& |[\Delta]| x: V: E_{0}|C \Rightarrow E| P(\bar{v})
\end{aligned}
$$

Fig. 1. $\mathrm{EB}^{3}$ syntax

the constructed data types and pattern-matching mechanisms of LNT enabled a natural description of $\mathrm{EB}^{3}$ data types and attribute functions.

We implemented our translation in the $\mathrm{EB}^{3} 2 \mathrm{LNT}$ tool, thus making possible the analysis of $\mathrm{EB}^{3}$ specifications using all the state-of-the-art features of the CADP toolbox, in particular the verification of data-based temporal properties expressed in MCL [18] using the on-the-fly model checker EVALUATOR 4.0.

The paper is organized as follows. Sections 2 and 3 give an overview of the $\mathrm{EB}^{3}$ and LNT languages, respectively. Section 4 presents our translation from $\mathrm{EB}^{3}$ to LNT, implemented by the $\mathrm{EB}^{3} 2 \mathrm{LNT}$ translator. Section 5 shows how $\mathrm{EB}^{3} 2 \mathrm{LNT}$ and CADP can be used for verifying the correctness requirements of an IS. Finally, Section 6 summarizes the results and draws up lines for future work.

\section{The Language $\mathrm{EB}^{3}$}

The $\mathrm{EB}^{3}$ method has been specially designed to specify the functional behaviour of ISs. A standard $\mathrm{EB}^{3}$ specification comprises (1) a class diagram representing entity types and associations for the IS being specified, (2) a process algebra specification, denoted by main, describing the IS, i.e., the valid traces of execution describing its behaviour, (3) a set of attribute function definitions, which are recursive functions on the system execution trace, and (4) input/output rules to specify outputs for input traces, or SQL expressions used to specify queries on the class diagram. We limit the presentation to the process algebra and the set of attribute functions. The $\mathrm{EB}^{3}$ syntax is presented in Figure 1 and the $\mathrm{EB}^{3}$ trace semantics $\mathrm{Sem}_{\mathrm{T}}[10]$ are given in Figure 2 as a set of rules named $\mathrm{T}_{1}$ to $\mathrm{T}_{11}$. Both figures are commented below.

Process expressions. We write $x, y, x_{1}, x_{2}, \ldots$ for variables and $v, w, v_{1}, v_{2}, \ldots$ for data expressions over user-defined domains, such as integers, Booleans and more complex domains that we do not give formally, for conciseness. Expressions are built over variables, constants, and standard operations. We also use the overlined notation as a shorthand notation for lists, e.g., $\bar{x}$ denotes a list of variables $x_{1}, \ldots, x_{n}$ of arbitrary length. An $\mathrm{EB}^{3}$ specification consists of a set of attribute function definitions $A_{1}, \ldots, A_{n}$, and of a set of process definitions of the form " $P(\bar{x})=E$ ", where $P$ is a process name and $E$ is a process expression.

Let Act be a set of actions written $\rho, \rho_{1}, \rho_{2}, \ldots$ and $L a b$ be a set of labels written $\alpha, \alpha_{1}, \alpha_{2}, \ldots$ Each action $\rho$ is either the internal action written $\lambda$, or a 


\begin{tabular}{|ll|}
\hline$\left(\mathrm{T}_{1}\right) \frac{\rho}{\rho \stackrel{\rho}{\rightarrow}}$ & $\left(\mathrm{T}_{7}\right) \frac{E_{1} \stackrel{\rho}{\rightarrow} E_{1}^{\prime}}{E_{1}|[\Delta]| E_{2} \stackrel{\rho}{\rightarrow} E_{1}^{\prime}|[\Delta]| E_{2}^{\prime}}$ in $(\rho, \Delta)$ \\
$\left(\mathrm{T}_{2}\right) \frac{E_{1} \stackrel{\rho}{\rightarrow} E_{1}^{\prime}}{E_{1} \cdot E_{2} \stackrel{\rho}{\rightarrow} E_{1}^{\prime} \cdot E_{2}}$ & $\left(\mathrm{~T}_{8}\right) \frac{E_{1} \stackrel{\rho}{\rightarrow} E_{1}^{\prime}}{E_{1}|[\Delta]| E_{2} \stackrel{\rho}{\rightarrow} E_{1}^{\prime}|[\Delta]| E_{2}} \neg$ in $(\rho, \Delta)$ \\
$\left(\mathrm{T}_{3}\right) \frac{E_{2} \stackrel{\rho}{\rightarrow} E_{2}^{\prime}}{\sqrt{ } \cdot E_{2} \stackrel{\rho}{\rightarrow} E_{2}^{\prime}}$ & $\left(\mathrm{T}_{9}\right) \frac{}{\sqrt{ }|[\Delta]| \sqrt{ } \stackrel{\lambda}{\rightarrow} \sqrt{ }}$ \\
$\left(\mathrm{T}_{4}\right) \frac{E_{1} \stackrel{\rho}{\rightarrow} E_{1}^{\prime}}{E_{1} \mid E_{2} \stackrel{\rho}{\rightarrow} E_{1}^{\prime}}$ & $\left(\mathrm{T}_{10}\right) \frac{E_{0} \stackrel{\rho}{\rightarrow} E_{0}^{\prime}}{C \rightarrow E_{0} \stackrel{\rho}{\rightarrow} E_{0}^{\prime}}\|C\|$ \\
$\left(\mathrm{T}_{5}\right) \frac{}{E_{0}{ }^{*} \stackrel{\lambda}{\rightarrow} \sqrt{ }}$ & $\left(\mathrm{T}_{11}\right) \frac{E[\bar{x}:=\bar{v}] \stackrel{\rho}{\rightarrow} E^{\prime}}{P(\bar{v}) \stackrel{\rho}{\rightarrow} E^{\prime}} P(\bar{x})=E$ \\
$\left(\mathrm{~T}_{6}\right) \frac{E_{0} \stackrel{\rho}{\rightarrow} E_{0}^{\prime}}{E_{0}{ }^{*} \stackrel{\rho}{\rightarrow} E_{0}^{\prime} \cdot E_{0}^{*}}$ &
\end{tabular}

Fig. 2. $\mathrm{EB}^{3}$ trace semantics $S e m_{\mathrm{T}}$

visible action of the form " $\alpha(\bar{v})$ ", where $\alpha \in L a b$. An action $\rho$ is the simplest process expression, whose semantics are given by rule $T_{1}$. The symbol $\sqrt{ }$ (which is not part of the user syntax) denotes successful execution. The trace $\mathrm{T}$ (implicit in the presentation) of an $\mathrm{EB}^{3}$ specification at a given moment consists of the sequence of visible actions executed since the start of the system. (Note therefore that $\lambda$ does not appear in the trace.) At system start, the trace is empty. If $\mathrm{T}$ denotes the current trace and action $\rho$ can be executed, then T. $\rho$ denotes the trace just after executing $\rho$.

$\mathrm{EB}^{3}$ processes can be combined with classical process algebra operators such as the sequence " $E_{1} \cdot E_{2}$ " $\left(\mathrm{T}_{2}, \mathrm{~T}_{3}\right)$, the choice " $E_{1} \mid E_{2}$ " $\left(\mathrm{T}_{4}\right)$ and the Kleene closure " $E_{0}{ }^{*}$ " $\left(\mathrm{T}_{5}, \mathrm{~T}_{6}\right)$. Rules $\left(\mathrm{T}_{7}\right.$ to $\left.\mathrm{T}_{9}\right)$ define parallel composition " $E_{1}|[\Delta]| E_{2}$ " of $E_{1}, E_{2}$ with synchronization on $\Delta \subseteq L a b$. The condition "in $(\rho, \Delta)$ " is true iff the label of $\rho$ belongs to $\Delta$. The symmetric rules for choice and parallel composition have been omitted for brevity. Expressions " $E_{1} \| \mid E_{2}$ " and " $E_{1} \| E_{2}$ " are equivalent respectively to " $E_{1}|[\emptyset]| E_{2}$ " and " $E_{1}|[L a b]| E_{2}$ ".

The guarded expression process " $C \Rightarrow E_{0}$ " $\left(\mathrm{T}_{10}\right)$ can execute $E_{0}$ if the Boolean condition $C$ holds, which is denoted by the side condition " $\|C\|$ ". Since $C$ may contain calls to attribute functions, its evaluation depends on the trace obtained up to the moment when the condition is evaluated. Note that the evaluation of the guard $C$ and the execution of the first action $\rho$ in $E_{0}$ are simultaneous, i.e., no action is allowed in concurrent processes in the meantime. We call this property the guard-action atomicity. This property is essential for consistency as, by side effects, the occurrence of actions in concurrent processes could implicitly change the value of $C$ before the guarded action has been executed. 
Quantification is permitted for choice and parallel composition. If $V$ is a set of expressions $\left\{v_{1}, \ldots, v_{n}\right\}, " \mid x: V: E_{0}$ " and "| $[\Delta] \mid x: V: E_{0}$ " stand respectively for " $E_{0}\left[x:=v_{1}\right]|\ldots| E_{0}\left[x:=v_{n}\right]$ " and " $E_{0}\left[x:=v_{1}\right]|[\Delta]| \ldots|[\Delta]| E_{0}\left[x:=v_{n}\right]$ ", where " $E[x:=v]$ " denotes the replacement of all occurrences of $x$ by $v$ in $E$. For instance, "\| $x:\{1,2,3\}: a(x)$ " stands for " $a(1)\|a(2)\| a(3)$ ". At last, named processes can be instantiated as usual $\left(\mathrm{T}_{11}\right)$. Given an $\mathrm{EB}^{3}$ process expression $E$, we write $\operatorname{vars}(E)$ for the set of variables occurring free in $E$.

Attribute functions. Attribute function definitions are denoted by the symbol $A$ in the grammar of Figure 1. Attribute functions are defined recursively on the current trace $T$ representing the history of actions executed, with the aid of functions last $(\mathrm{T})$ which denotes the last action of the trace, and front $(\mathrm{T})$ which denotes the trace without its last action. The symbol $\perp$ represents the undefined value. In particular, both last $(\mathrm{T})$ and front $(\mathrm{T})$ match $\perp$ when the trace is empty. The symbol _ (wildcard) matches all actions not matched by any of the preceding action patterns $\alpha_{1}\left(\overline{x_{1}}\right), \ldots, \alpha_{q}\left(\overline{x_{q}}\right)$. Each $v_{i}(i \in 0 . . n)$ is an expression of the same type as $f$ 's return type built over the variables $\bar{y} \cup \overline{x_{i}}$.

For defining formal semantics for attribute functions, the rule system of Figure 2 has to be expanded with trace and memory contexts for each process, representing the sequence of actions executed since the process was initiated, and the value of attribute functions for the current trace and any value for the rest of their arguments, stored into process memory M. Due to space limitations, we do not present the formal semantics here, but show how attribute functions are evaluated on a concrete example. The formal trace-memory semantics for attribute functions can be found in the companion paper [20].

Example. We give an example of how the trace-memory semantics work for a simplified library management system, whose specification (processes and attribute functions) in $\mathrm{EB}^{3}$ is given in Figure 3. Process main is the parallel interleaving between $m$ instances of process book and $p$ instances of process member. Process book stands for a book acquisition followed by its eventual discard. The attribute function "borrower $(\mathrm{T}, b I d)$ " looks for actions of the form "Lend (mId, bId)" or "Return (bId)" in the trace and returns the current borrower of book bId or $\perp$ if the book is not lent. In process book, action "Discard (bId)" is thus guarded to guarantee that book bId cannot be discarded if it is currently lent. How the use of attribute functions enhances expressiveness in the $\mathrm{EB}^{3}$ specification of Figure 3 is discussed in [20].

We illustrate how the $\mathrm{EB}^{3}$ specification describing the library management system is evaluated. The idea lies in the observation that attribute functions can be turned into state variables (the memory $\mathrm{M}$ ) carrying the effect of the system trace on their corresponding values. This avoids keeping the (ever-growing) trace leading to a finite state model. If $f\left(\mathrm{~T}, x_{1}: T_{1}, \ldots, x_{l}: T_{l}\right)$ is an attribute function, we construct $\left|T_{1}\right| \times \ldots \times\left|T_{l}\right|$ state variables, where $\left|T_{i}\right|(i \in 1 . . l)$ stands for $T_{i}$ 's cardinality.

As an example, we set $m=p=N b$ Loans $=2$, i.e. we consider two books $b_{1}$ and $b_{2}$, and two members $m_{1}$ and $m_{2}$. The memory has four cells: $\mathbf{M}=$ 


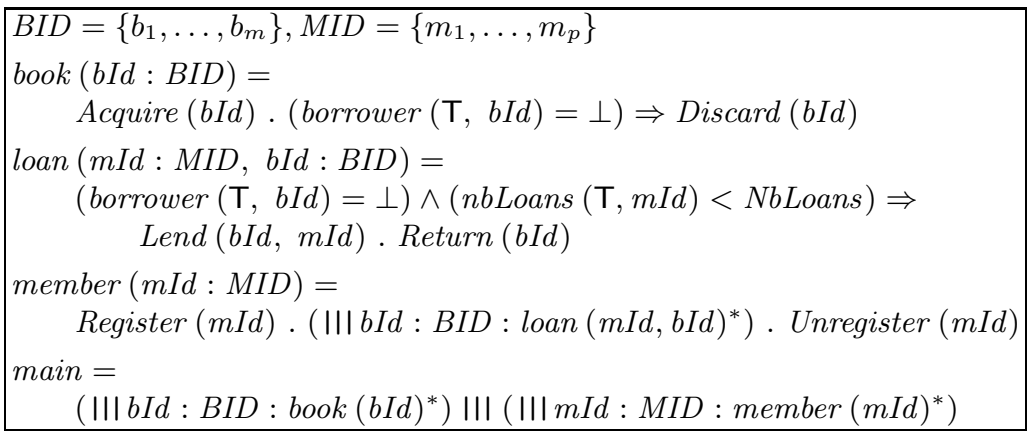

\begin{tabular}{|c|c|}
\hline $\begin{array}{l}\text { nbLoans }(\mathrm{T}: \mathcal{T}, m I d: M I D): N_{\perp}= \\
\text { match last }(\mathrm{T}) \text { with } \\
\quad \perp: \perp \\
\text { | Lend }(\text { bId }, m I d): \\
\quad \text { nbLoans }(\text { front }(\mathrm{T}), m I d)+1 \\
\text { | Register }(m I d): 0 \\
\text { | Unregister }(m I d): \perp \\
\text { | Return }(\text { bId }): \\
\quad \text { if } m I d=\text { borrower }(\mathrm{T}, b I d) \text { then } \\
\quad n b L o a n s(\text { front }(\mathrm{T}), m I d)-1 \\
\quad \text { else nbLoans }(\text { front }(\mathrm{T}), m I d) \text { end if } \\
\text { | _: nbLoans }(\text { front }(\mathrm{T}), m I d) \\
\text { end match }\end{array}$ & $\begin{array}{l}\text { borrower }(\mathrm{T}: \mathcal{T}, \text { bId }: B I D): M I D_{\perp}= \\
\quad \text { match last }(\mathrm{T}) \text { with } \\
\quad \perp: \perp \\
\text { | Lend }(\text { bId }, m I d): m I d \\
\text { | Return }(\text { bId }): \perp \\
\text { | _: borrower }(\text { front }(\mathrm{T}), b I d) \\
\text { end match }\end{array}$ \\
\hline
\end{tabular}

Fig. 3. $\mathrm{EB}^{3}$ specification of a library management system

(borrower $\left[b_{1}\right]$, borrower $\left[b_{2}\right], n b$ Loans $\left[m_{1}\right], n b$ Loans $\left.\left[m_{2}\right]\right)$. The first two cells keep the two values of the attribute function $\operatorname{borrower}(\mathrm{T}, \bullet)$ for a given trace $\mathrm{T}$, and the last two keep the values of nbLoans $(\mathrm{T}, \bullet)$. After every step, the new value of each cell can be calculated from the previous memory and the action that has just been executed. The memory is initially set to $(\perp, \perp, \perp, \perp)$. After the trace "Acquire $\left(b_{1}\right)$.Acquire $\left(b_{2}\right)$.Register $\left(m_{1}\right)$.Register $\left(m_{2}\right)$ " the memory contains $(\perp, \perp, 0,0)$. If action "Lend $\left(b_{1}, m_{1}\right)$ " is then executed, the new memory is $\left(m_{1}, \perp, 1,0\right)$. For instance, the new value $m_{1}$ for borrower $\left[b_{1}\right]$ is obtained from the rule "Lend $(b I d, m I d): m I d$ " in the definition of the attribute function borrower (see Fig. 3), and the new value 1 for $n b$ Loans $\left[m_{1}\right]$ by the rule "Lend $(b I d, m I d):$ nbLoans (front $(\mathrm{T}), m I d)+1$ " of the attribute function $n b$ Loans, where the value of nbLoans (front $(\mathrm{T}), m_{1}$ ) corresponds to the value of $n b$ Loans $\left[m_{1}\right]$ in the previous memory state (value 0 ).

\section{The Language LNT}

LNT aims at providing the best features of imperative and functional programming languages and value-passing process algebras. It has a user friendly syntax and formal operational semantics defined in terms of labeled transition systems 


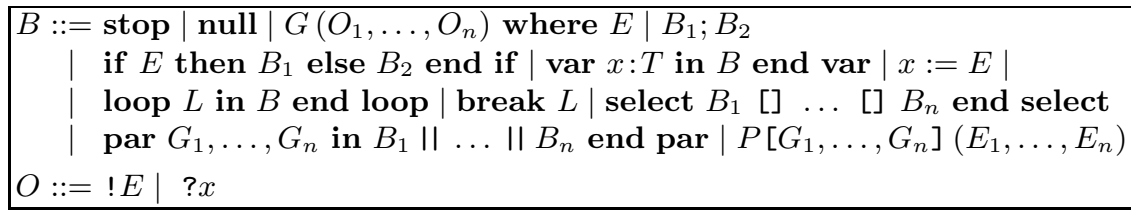

Fig. 4. LNT syntax (limited to the fragment used in this paper)

(LTSs). LNT is supported by the LNT.OPEN tool of CADP, which allows the on-the-fly exploration of the LTS corresponding to an LNT specification.

We present the fragment of LNT that serves as the target of our translation. Its syntax is given in Figure 4. LNT terms denoted by $B$ are built from actions, choice (select), conditional (if), sequential composition (;), breakable loop (loop and break) and parallel composition (par). Communication is carried out by rendezvous on gates, written $G, G_{1}, \ldots, G_{n}$, and may be guarded using Boolean conditions on the received values (where clause). LNT allows multiway rendezvous with bidirectional (send/receive) value exchange on the same gate occurrence, each offer $O$ being either a send offer (!) or a receive offer (?), independently of the other offers. Expressions $E$ are built from variables, type constructors, function applications and constants. Labels $L$ identify loops, which can be escaped using "break $L$ " from inside the loop body. Processes are parameterized by gates and data variables. LNT semantics are formally defined in SOS style in [7].

\section{Translation from $\mathrm{EB}^{3}$ to LNT}

Principles. Our translation of $\mathrm{EB}^{3}$ relies on the trace-memory semantics. Thus, we explicitly model in LNT a memory, which stores the state variables corresponding to attribute functions (we call these variables attribute variables) and is modified each time an action is executed.

Assuming $n$ attribute functions $f_{1}, \ldots, f_{n}$, we model the memory as a process $M$ placed in parallel with the rest of the system (a common approach for modeling global variables in process algebras), which manages for each attribute function $f_{i}$ an attribute variable (also named $f_{i}$ ) that encodes the function. To read the values of these attribute variables (i.e., to evaluate the attribute functions), processes need to communicate with the memory $M$, and every action must have an immediate effect on the memory (so as to reflect the immediate effect on the execution trace). To achieve this, the memory process synchronizes with the rest of the system on every possible action of the system (including $\lambda$, to which we associate an LNT gate also written $\lambda$ in abstract syntax for convenience), and updates its attribute variables accordingly. The list of attribute variables $\bar{f}=\left(f_{1}, \ldots, f_{n}\right)$ is added as a supplementary offer on each $\mathrm{EB}^{3}$ action $\alpha(\bar{v})$, so that attribute variables can be directly accessed to evaluate the guard associated to the action, wherever needed, while guaranteeing the guard-action 
atomicity. Therefore, every action $\alpha(\bar{v})$ will be encoded in LNT as $\alpha(! \bar{v}, ? \bar{f})$, and synchronized with an action of the form $\alpha(? \bar{x}, ! \bar{f})$ in the memory process $M$, thus taking benefit of bidirectional value exchange during the rendezvous.

Translation of attribute functions. To formalize the translation, we assume $L a b=$ $\left\{\alpha_{1}, \ldots, \alpha_{q}\right\}$ (not including $\lambda$ ), each $\alpha_{j}$ has formal parameters $\overline{x_{j}},\left\{f_{1}, \ldots, f_{n}\right\}$ is the set of attribute functions, and each $f_{i}$ is uniquely defined by the set of formal parameters $\overline{y_{i}}$ and the set of data expressions $w_{i}^{0}, \ldots, w_{i}^{q}$, such that:

$$
f_{i}\left(\mathbf{T}, \overline{y_{i}}\right)=\text { match last }(\mathbf{T}) \text { with } \perp: w_{i}^{0}\left|\alpha_{1}\left(\overline{x_{1}}\right): w_{i}^{1}\right| \ldots \mid \alpha_{q}\left(\overline{x_{q}}\right): w_{i}^{q}
$$

We also assume that the attribute functions are ordered, so that for all $h \in$ $1 . . n, i \in 1 . . n, j \in 1 . . q$, every function call of the form $f_{h}(\mathrm{~T}, \ldots)$ occurring in $w_{i}^{j}$ satisfies $h<i$ and every call of the form $f_{h}($ front $(\mathrm{T}), \ldots)$ satisfies $h \geq i$. Such an ordering can be constructed if the $\mathrm{EB}^{3}$ specification does not contain circular dependencies between function calls, which would potentially lead to infinite attribute function evaluation. In particular, the definition of an attribute function $f_{i}$ cannot contain recursive calls of the form " $f_{i}(\mathrm{~T}, \ldots)$ ", but only recursive calls of the form " $f_{i}$ (front $\left.(\mathbf{T}), \ldots\right)$ ". Note that this does not limit the expressiveness of $\mathrm{EB}^{3}$ attribute functions, because every recursive computation on data expressions only (which keeps the trace unchanged) can be described using standard functions and not attribute functions.

Ordering attribute functions in this way allows the memory to be updated consistently, from $f_{1}$ to $f_{n}$ in turn. At every instant, already-updated values correspond to calls of the form $f_{h}(\mathrm{~T}, \ldots)$ (the value of $f_{h}$ on the current trace), whereas calls of the form $f_{h}$ (front $(\mathrm{T}), \ldots$ ) are replaced by accesses to a copy $\overline{f^{\prime}}$ of the memory $\bar{f}$, which was made before starting the update. This encoding thus enables the trace parameter to be discharged from function calls, ensuring that while updating $f_{i}$, accesses to $f_{h}$ with $h<i$ necessarily correspond to calls with parameter $\mathrm{T}$.

Process $M$ is defined in Figure 5. It runs an infinite loop, which "listens" to all possible actions $\alpha_{j}$ of the system. Each attribute variable $f_{i}$ is an array with $l_{i}$ dimensions, where $l_{i}$ is the arity of the attribute function $f_{i}$ minus 1 (because the trace parameter is now discharged). Each dimension of the array $f_{i}$ thus corresponds to one formal parameter in $\overline{y_{i}}$, so that $f_{i}\left[\operatorname{ord}\left(v_{1}\right)\right] \ldots\left[\operatorname{ord}\left(v_{l_{i}}\right)\right]$ encodes the current value of $f_{i}\left(\mathrm{~T}, v_{1}, \ldots, v_{l_{i}}\right)$, where ord $(v)$ is a predefined LNT function that denotes the ordinate of value $v$, i.e., a unique number between 1 and the cardinal of $v$ 's type. For each type $T$ we assume the existence of functions first $_{T}$ that returns the first element of type $T$, last $_{T}$ that returns the last element of type $T$, and $\operatorname{next}_{T}(x)$ that returns the successor of $x$ in type $T$ (following the total order induced by ord). Such functions are available in LNT for all finite types. Function mod transforms an expression $E$ by syntactically replacing function calls by array accesses, while discharging the trace parameter as explained above.

Upon synchronisation on action $\alpha_{j}\left(? \overline{x_{j}}, ! \bar{f}\right)$ with the LNT process corresponding to $\mathrm{EB}^{3}$ 's main process (see translation of processes below), the values of all attribute variables $f_{i}(i \in 1 . . n)$ are updated using function $u p d_{i}^{j}$. 


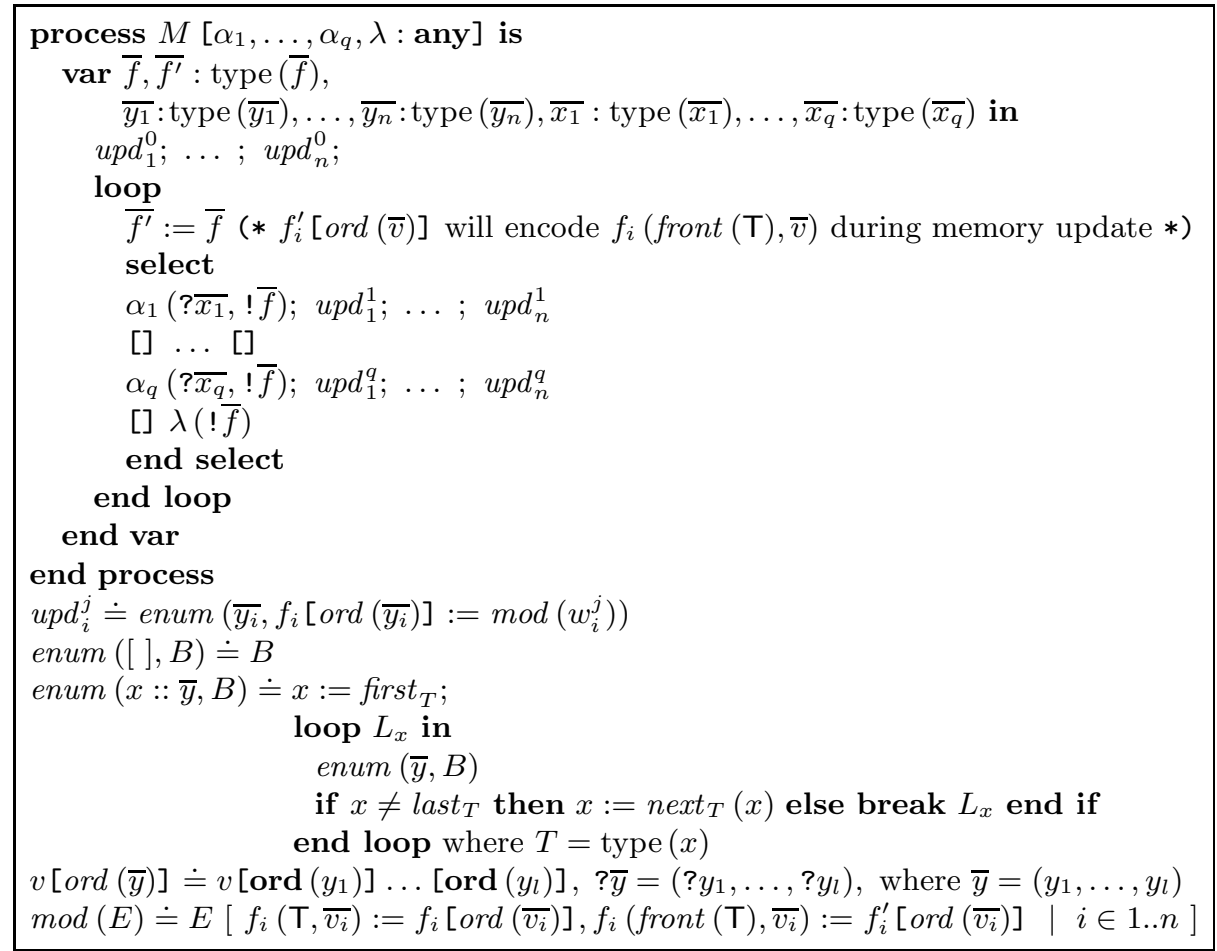

Fig. 5. LNT code for the memory process implementing attribute functions

Translation of processes. We define a translation function $t$ from an $\mathrm{EB}^{3}$ process expression to an LNT process. Most $\mathrm{EB}^{3}$ constructs are process algebra constructs with a direct correspondence in LNT. The main difficulty arises in the translation of guarded process expressions of the form " $C \Rightarrow E_{0}$ " in a way that guarantees the guard-action atomicity. This led us to consider a second parameter for the translation function $t$, namely the condition $C$, whose evaluation is delayed until the first action occurring in the process expression $E_{0}$. The definition of $t(E, C)$ is given in Figure 6 . An $\mathrm{EB}^{3}$ specification $E_{0}$ will then be translated into "par $\alpha_{1}, \ldots, \alpha_{q}, \lambda$ in $t\left(E_{0}\right.$, true $) \| M\left[\alpha_{1}, \ldots, \alpha_{q}, \lambda\right]$ end par" and every process definition of the form " $P(\bar{x})=E$ " will be translated into the process "process $P\left[\alpha_{1}, \ldots, \alpha_{q}, \lambda\right.$ : any $](\bar{x}:$ type $(\bar{x}))$ is $t(E$, true) end process", where $\left\{\alpha_{1}, \ldots, \alpha_{q}\right\}=L a b$. The rules of Figure 6 can be commented as follows:

- Rule (1) translates the $\lambda$ action. Note that $\lambda$ cannot be translated to the empty LNT statement null, because execution of $\lambda$ may depend on a guard $C$, whose evaluation requires the memory to be read, so as to get attribute variable values. This is done by the LNT communication action $\lambda(? \bar{f})$. The guard $C$ is evaluated after replacing calls to attribute functions (all of which have the form $f_{i}\left(\mathrm{~T}, \bar{v}_{i}\right)$ ) by the appropriate attribute variables, using function mod defined in Figure 5. Rule (2) is similar but handles visible actions. 


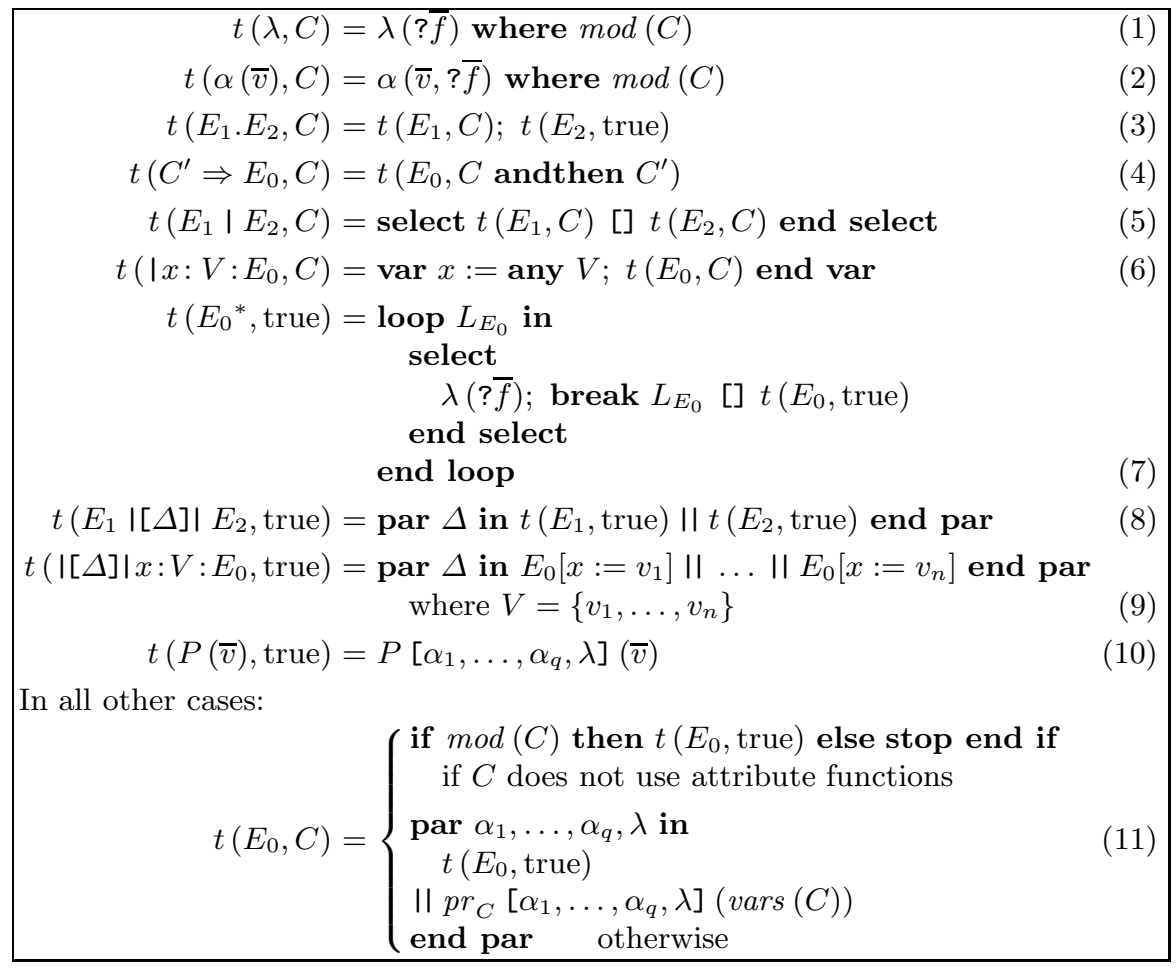

Fig. 6. Translation from $\mathrm{EB}^{3}$ process to LNT process

- Rule (3) translates $\mathrm{EB}^{3}$ sequential composition into LNT sequential composition, passing the evaluation of $C$ to the first process expression.

- Rule (4) makes a conjunction between the guard of the current process expression with the guard already accumulated from the context.

- Rules (5) and (6) translate the choice and quantified choice operators of $\mathrm{EB}^{3}$ into their direct LNT counterpart.

- Rule (7) translates the Kleene closure into a combination of LNT loop and select, following the identity $E_{0}{ }^{*}=\lambda \mid E_{0} \cdot E_{0}{ }^{*}$.

- Rule (8) translates $\mathrm{EB}^{3}$ parallel composition into LNT parallel composition.

- Rule (9) translates $\mathrm{EB}^{3}$ quantified parallel composition into LNT parallel composition by expanding the type $V$ of the quantification variable, since LNT does not have a quantified parallel composition operator.

- Rule (10) translates an $\mathrm{EB}^{3}$ process call into the corresponding LNT process call, which requires gates to be passed as parameters.

- Rules (7) to (10) only apply when the guard $C$ is trivially true. In the other cases, we must apply rule (11), which generates code implementing the guard. If $C$ does not use attribute functions, i.e., does not depend on the trace, then it can be evaluated immediately without communicating with the memory 
process (first case). Otherwise, the guard evaluation must be delayed until the first action of the process expression $E_{0}$. When $E_{0}$ is either a Kleene closure, a parallel composition, or a process call, identifying its first action syntactically is not obvious. One solution would consist in expanding $E_{0}$ into a choice in which every branch has a fixed initial action ${ }^{3}$, to which the guard would be added. We preferred an alternative solution that avoids the potential combinatorial explosion of code due to static expansion. A process $p r_{C}$ (defined in Fig. 7) is placed in parallel to $t\left(E_{0}\right.$, true) and both processes synchronize on all actions. Process $p r_{C}$ imposes on $t\left(E_{0}\right.$, true) the constraint that the first executed action must satisfy the condition $C$ (then branch). For subsequent actions, the condition is relaxed (else branch).

The following example illustrates and justifies the use of process $p r_{C}$ as a means to solve the guard-action atomicity problem. Consider the $\mathrm{EB}^{3}$ system " $C \Rightarrow \operatorname{Lend}\left(b_{1}, m_{1}\right)$ III Return $\left(b_{2}\right)$ ", where $C$ denotes the Boolean condition "borrower $\left(\mathrm{T}, b_{1}\right)=\perp \wedge$ nbLoans $\left(\mathrm{T}, m_{1}\right)<N b$ Loans" and Lab $=$ $\{$ Lend, Return $\}$. The LNT code corresponding to this system is the following:

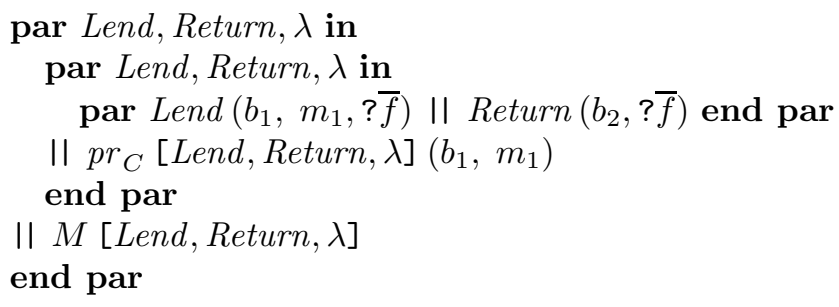

The first action executed by this system may be either Lend or Return. We consider the case where Lend is executed first. According to the LNT semantics, it results from the multiway synchronization of the following three actions:

- "Lend $\left(b_{1}, m_{1}, ? \bar{f}\right)$ " in the above expression,

- "Lend $(? b, ? m, ? \bar{f})$ where borrower $\left[\operatorname{ord}\left(b_{1}\right)\right]=\perp \wedge n b \operatorname{Loans}\left[\operatorname{ord}\left(m_{1}\right)\right]<$ NbLoans" in process $p r_{C}$ (at this moment, start is true, see Fig. 7), and

- "Lend $(? b, ? m, ! \bar{f})$ " in process $M$ (see Fig. 5).

Thus, in $p r_{C}$ at synchronization time, $\bar{f}$ is an up-to-date copy of the memory stored in $M, b=b_{1}$, and $m=m_{1}$. The only condition for the synchronization to occur is the guard $\bmod (C)$, whose value is computed using the up-to-date copy $\bar{f}$ of the memory. In case $\bmod (C)$ evaluates to true, no other action (susceptible to modifying $\bar{f}$ ) can occur between the evaluation of $\bmod (C)$ and the occurrence of Lend as both happen synchronously, thus achieving the guard-action atomicity. Once Lend has occurred, Return can occur without any condition, as the value of start has now become false.

${ }^{3}$ Such a form, commonly called head normal form [3], is used principally in the context of the process algebra ACP [4] to analyse the behaviour of recursive processes. 


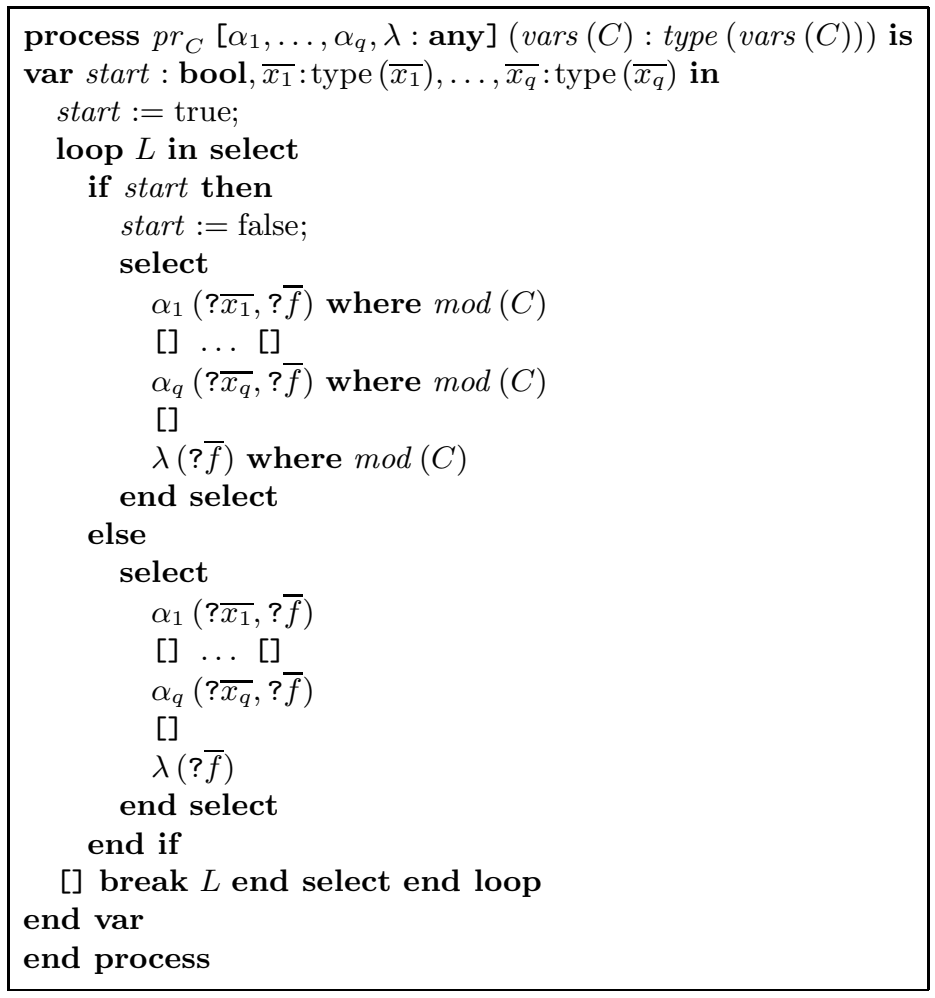

Fig. 7. Process $p r_{C}$

Theorem 1. Let $E, E^{\prime}$ be $\mathrm{EB}^{3}$ process expressions, $\mathrm{T}$ be the current trace, $\bar{f}$ be the set of attribute functions, and $\rho \in$ Act. Then $E \stackrel{\rho(\bar{x})}{\longrightarrow} E^{\prime}$ if and only if:

$$
t(E, \text { true }) \stackrel{\rho(\bar{x}, \bar{f})}{\longrightarrow} t\left(E^{\prime}, \text { true }\right) \wedge\left(\forall f_{i} \in \bar{f}\right)(\forall \bar{v}) f_{i}(\mathrm{~T}, \bar{v})=f_{i}[\operatorname{ord}(\bar{v})] .
$$

The proof strategy for Theorem 1 relies on the existence of a bisimulation between each $\mathrm{EB}^{3}$ specification and its corresponding LNT translation. It works by providing a match between the reduction rules of $\mathrm{EB}^{3}[20]$ and the corresponding LNT rules [7].

We developed an automatic translator tool from $\mathrm{EB}^{3}$ specifications to LNT, named $\mathrm{EB}^{3}$ 2LNT, implemented using the Ocaml Lex/Yacc compiler construction technology. It consists of about 900 lines of OCaml code. We applied EB ${ }^{3} 2 \mathrm{LNT}$ on a benchmark of $\mathrm{EB}^{3}$ specifications, which includes variations of the library management system (examined in its simplest version in Section 2) and a bank account management system.

We noticed that, for each $\mathrm{EB}^{3}$ specification, the code size of the equivalent LNT specification is twice as big. Part of this expansion is caused by the fact that LNT is more structured than $\mathrm{EB}^{3}$ : LNT requires more keywords and gates 
have to be declared and passed as parameters to each process call. By looking at the rules of Figure 6, we can see that the other causes of expansion are rule (5), which duplicates the condition $C$, and rule (9), which duplicates the body $E_{0}$ of the quantified parallel composition operator " $|[\Delta]| x: V: E_{0}$ " as many times as there are elements in the set $V$. Both expansions are linear in the size of the source $\mathrm{EB}^{3}$ code. However, in the case of a nested parallel composition "|[ $\left.\Delta_{1}\right]\left|x_{1}: V_{1}: \ldots\right|\left[\Delta_{n}\right] \mid x_{n}: V_{n}: E_{0}$ ", the expansion factor is as high as the product of the number of elements in the respective sets $V_{1}, \ldots, V_{n}$, which may be large. If $E_{0}$ is a big process expression, the expansion can be limited by encapsulating $E_{0}$ in a parameterized process " $P_{E_{0}}\left(x_{1}, \ldots, x_{n}\right)$ " and replacing duplicated occurrences of $E_{0}$ by appropriate instances of $P_{E_{0}}$.

\section{Case Study}

We illustrate below the application of the $\mathrm{EB}^{3} 2 \mathrm{LNT}$ translator in conjunction with CADP for analyzing an extended version of the IS library management system, whose description in $\mathrm{EB}^{3}$ can be found in Annex $\mathrm{C}$ of [12]. With respect to the simplified version presented in Section 2, the IS enables e.g., members to renew their loans and to reserve books, and their reservations to be cancelled or transferred to other members on demand. The desired behaviour of this IS was characterized in [9] as a set of 15 requirements expressed informally as follows:

R1. A book can always be acquired by the library when it is not currently acquired.

R2. A book cannot be acquired by the library if it is already acquired.

R3. An acquired book can be discarded only if it is neither borrowed nor reserved.

R4. A person must be a member of the library in order to borrow a book.

R5. A book can be reserved only if it has been borrowed or already reserved by some member.

R6. A book cannot be reserved by the member who is borrowing it.

R7. A book cannot be reserved by a member who is reserving it.

R8. A book cannot be lent to a member if it is reserved.

R9. A member cannot renew a loan or give the book to another member if the book is reserved.

R10. A member is allowed to take a reserved book only if he owns the oldest reservation.

R11. A book can be taken only if it is not borrowed.

R12. A member who has reserved a book can cancel the reservation at anytime before he takes it.

R13. A member can relinquish library membership only when all his loans have been returned and all his reservations have either been used or cancelled.

R14. Ultimately, there is always a procedure that enables a member to leave the library.

R15. A member cannot borrow more than the loan limit defined at the system level for all users.

We expressed all the above requirements using the property specification language MCL [18]. MCL is an extension of the alternation-free modal $\mu$-calculus [8] with action predicates enabling value extraction, modalities containing extended regular expressions on transition sequences, quantified variables and parameterized fixed point operators, programming language constructs, and fairness 
operators encoding generalized Büchi automata. These features make possible a concise and intuitive description of safety, liveness, and fairness properties involving data, without sacrificing the efficiency of on-the-fly model checking, which has a linear-time complexity for the dataless MCL formulas [18].

We show below the MCL formulation of two requirements from the list above, which denote typical safety and liveness properties. Requirement R2 is expressed in MCL as follows:

[true*. $\{$ ACQUIRE ? $B$ : string $\}$. (not $\{$ DISCARD $! B\})^{*}$. $\{$ ACQUIRE $\left.! B\}\right]$ false

This formula uses the standard safety pattern "[ $\beta]$ false", which forbids the existence of transition sequences matching the regular formula $\beta$. Here the undesirable sequences are those containing two Acquire operations for the same book $B$ without a Discard operation for $B$ in the meantime. The regular formula true* matches a subsequence of (zero or more) transitions labeled by arbitrary actions. Note the use of the construct "? $B$ : string", which matches any string and extracts its value in the variable $B$ used later in the formula. Therefore, the above formula captures all occurrences of books carried by Acquire operations in the model. Requirement R12 is formulated in MCL as follows:

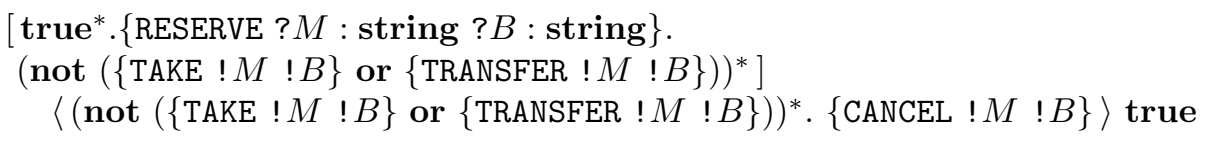

This formula denotes a liveness property of the form "[ $\left.\beta_{1}\right]\left\langle\beta_{2}\right\rangle$ true", which states that every transition sequence matching the regular formula $\beta_{1}$ (in this case, book $B$ has been reserved by member $M$ and subsequently neither taken nor transferred) ends in a state from which there exists a transition sequence matching the regular formula $\beta_{2}$ (in this case, the reservation can be cancelled before being taken or transferred).

Using $\mathrm{EB}^{3} 2 \mathrm{LNT}$, we translated the $\mathrm{EB}^{3}$ specification of the library management system to LNT. The resulting specification was checked against all the 15 requirements, formulated in MCL, using the EVALUATOR 4.0 model checker of CADP. The experiments were performed on an Intel(R) Core(TM) i7 CPU 880 at $3.07 \mathrm{GHz}$. Table 1 shows the results for several configurations of the IS, obtained by instantiating the number of books $(m)$ and members $(p)$ in the IS. All requirements were shown to be valid on the IS specification. The second and third line of the table indicate the number of states and transitions of the LTS corresponding to the LNT specification. The fourth line gives the time needed to generate the LTS and the other lines give the verification time for each requirement. Note that the number of states generated increases with the size of $m$ and $p$ as EVALUATOR 4.0 applies explicit techniques for state space generation.

\section{Conclusion}

We proposed an approach for equipping the $\mathrm{EB}^{3}$ method with formal verification capabilities by reusing already available model checking technology. Our 
Table 1. Model checking results for the library management system

\begin{tabular}{|r|r|r|r|r|}
\hline$(m, p)$ & $(3,2)$ & $(3,3)$ & $(3,4)$ & $(4,3)$ \\
\hline states & 1,002 & 182,266 & $8,269,754$ & $27,204,016$ \\
\hline trans. & 5,732 & $1,782,348$ & $105,481,364$ & $330,988,232$ \\
\hline time & $1.9 \mathrm{~s}$ & $14.4 \mathrm{~s}$ & $31^{\prime} 39 \mathrm{~s}$ & $140^{\prime} 22 \mathrm{~s}$ \\
\hline \hline R1 & $0.3 \mathrm{~s}$ & $1.8 \mathrm{~s}$ & $5^{\prime} 19 \mathrm{~s}$ & $20^{\prime} 13 \mathrm{~s}$ \\
\hline R2 & $0.2 \mathrm{~s}$ & $2.9 \mathrm{~s}$ & $9^{\prime} 26 \mathrm{~s}$ & $36^{\prime} 7 \mathrm{~s}$ \\
\hline R3 & $0.2 \mathrm{~s}$ & $9.4 \mathrm{~s}$ & $97^{\prime} 46 \mathrm{~s}$ & $26^{\prime} 47 \mathrm{~s}$ \\
\hline R4 & $0.2 \mathrm{~s}$ & $1.7 \mathrm{~s}$ & $5^{\prime} 15 \mathrm{~s}$ & $18^{\prime} 40 \mathrm{~s}$ \\
\hline R5 & $0.2 \mathrm{~s}$ & $2.2 \mathrm{~s}$ & $6^{\prime} 46 \mathrm{~s}$ & $21^{\prime} 52 \mathrm{~s}$ \\
\hline R6 & $0.2 \mathrm{~s}$ & $4.1 \mathrm{~s}$ & $38^{\prime} 30 \mathrm{~s}$ & $10^{\prime} 19 \mathrm{~s}$ \\
\hline R7 & $0.2 \mathrm{~s}$ & $7.4 \mathrm{~s}$ & $65^{\prime} 22 \mathrm{~s}$ & $24^{\prime} 33 \mathrm{~s}$ \\
\hline R8 & $0.2 \mathrm{~s}$ & $2.2 \mathrm{~s}$ & $6^{\prime} 52 \mathrm{~s}$ & $22^{\prime} 27 \mathrm{~s}$ \\
\hline R9 & $0.2 \mathrm{~s}$ & $2.3 \mathrm{~s}$ & $6^{\prime} 38 \mathrm{~s}$ & $22^{\prime} 29 \mathrm{~s}$ \\
\hline R10 & $0.3 \mathrm{~s}$ & $13.3 \mathrm{~s}$ & $43^{\prime} 59 \mathrm{~s}$ & $62^{\prime} 07 \mathrm{~s}$ \\
\hline R11 & $0.3 \mathrm{~s}$ & $2.5 \mathrm{~s}$ & $6^{\prime} 36 \mathrm{~s}$ & $22^{\prime} 14 \mathrm{~s}$ \\
\hline R12 & $0.3 \mathrm{~s}$ & $4.0 \mathrm{~s}$ & $10^{\prime} 47 \mathrm{~s}$ & $45^{\prime} 09 \mathrm{~s}$ \\
\hline R13 & $0.4 \mathrm{~s}$ & $4.3 \mathrm{~s}$ & $11^{\prime} 46 \mathrm{~s}$ & $1^{\prime} 07 \mathrm{~s}$ \\
\hline R14 & $0.3 \mathrm{~s}$ & $3.6 \mathrm{~s}$ & $10^{\prime} 41 \mathrm{~s}$ & $37^{\prime} 33 \mathrm{~s}$ \\
\hline R15 & $0.2 \mathrm{~s}$ & $2.8 \mathrm{~s}$ & $7^{\prime} 53 \mathrm{~s}$ & $28^{\prime} 56 \mathrm{~s}$ \\
\hline
\end{tabular}

approach relies upon a new translation from $\mathrm{EB}^{3}$ to $\mathrm{LNT}$, which provides a direct connection to all the state-of-the-art verification features of the CADP toolbox. The translation, based on alternative memory semantics of $\mathrm{EB}^{3}[20]$ instead of the original trace semantics [10], was automated by the $\mathrm{EB}^{3} 2 \mathrm{LNT}$ translator and validated on several examples of typical ISs. So far, we experimented only the model checking of MCL data-based temporal properties on $\mathrm{EB}^{3}$ specifications. However, CADP also provides extensive support for equivalence checking and compositional LTS construction, which can be of interest to IS designers.

As future work, we plan to provide a formal proof of the translation from $\mathrm{EB}^{3}$ to LNT, which could serve as reference for translating $\mathrm{EB}^{3}$ to other process algebras as well. We also plan to study abstraction techniques for verifying properties regardless of the number of entity instances that participate in the IS, following the approaches for parameterized model checking [1]. In particular, we will observe how the insertion of new functionalities into an IS affects this issue, and we will formalize this in the context of $\mathrm{EB}^{3}$ specifications.

\section{References}

1. P.A. Abdulla, A. Bouajjani, B. Jonsson, M. Nilsson. Handling Global Conditions in Parameterized System Verification. In Proc. CAV, LNCS vol. 1633, pages 134-145, Springer, 1999.

2. J.-R. Abrial. The B-Book - Assigning programs to meanings. Cambridge University Press, 2005.

3. J.A. Bergstra, A. Ponse, S.A. Smolka. Handbook of Process Algebra. Elsevier, 2001. 
4. J.A. Bergstra, J. W. Klop. Algebra of Communicating Processes with Abstraction. TCS, 37:77-121, 1985.

5. R. Chossart. Évaluation d'outils de vérification pour les spécifications de systèmes d'information. Master's thesis, Université de Sherbrooke, 2010.

6. ClearSy. Atelier B. http://www.atelierb.societe.com.

7. D. Champelovier, X. Clerc, H. Garavel, Y. Guerte, C. McKinty, V. Powazny, F. Lang, W. Serwe, G. Smeding. Reference Manual of the LOTOS NT to LOTOS Translator - Version 5.4. INRIA/VASY, 2011.

8. E. Allen Emerson, C-L. Lei. Efficient Model Checking in Fragments of the Propositional Mu-Calculus. In Proc. of LICS, pages 267-278, 1986.

9. M. Frappier, B. Fraikin, R. Chossart, R. Chane-Yack-Fa, M. Ouenzar. Comparison of model checking tools for information systems. In Proc. of ICFEM, LNCS vol. 6447, pages 581-596, Springer, 2010.

10. M. Frappier, R. St.-Denis. EB $^{3}$ : an entity-based black-box specification method for information systems. Software and System Modeling 2(2):134-149, Springer, 2003.

11. H. Garavel, F. Lang, R. Mateescu, W. Serwe. CADP 2010: A toolbox for the construction and analysis of distributed processes. In Proc. of TACAS, LNCS vol. 6605, pages 372-387, Springer, 2011.

12. F. Gervais. Combinaison de spécifications formelles pour la modélisation des systèmes d'information. PhD thesis, Université de Sherbrooke, 2006.

13. F. Gervais, M. Frappier, R. Laleau. Synthesizing B Specifications from $\mathrm{EB}^{3}$ Attribute Definitions. In Proc. of iFM, LNCS vol. 3771, pages 207-226 Springer, 2005.

14. F. Gervais, M. Frappier, R. Laleau. Refinement of $\mathrm{EB}^{3}$ Process Patterns into B Specifications. In Proc. of Formal Specification and Development in B, LNCS vol. 4355, pages 201-215, Springer, 2006.

15. C. A. R. Hoare. Communicating Sequential Processes. Commun. ACM, 21(8):666677, 1978.

16. ISO/IEC. Enhancements to LOTOS (E-LOTOS). International Standard number 15437:2001, International Organization for Standardization - Information Technology, Genève, 2001.

17. M. E. Jiague, M. Frappier, F. Gervais, P. Konopacki, R. Laleau, J. Milhau, R. St-Denis. Model-Driven Engineering of Functional Security Policies. In Proc. of ICEIS, pages 374-379, 2010.

18. R. Mateescu, D. Thivolle. A model checking language for concurrent value-passing systems. In Proc. of FM, LNCS vol. 5014, pages 148-164, Springer, 2008.

19. J. Milhau, A. Idani, R. Laleau, M.A. Labiadh, Y. Ledru, M. Frappier. Combining UML, ASTD and B for the formal specification of an access control filter. In Journal of Innovations in Systems and Software Engineering, vol. 7, pages 303313, Springer, 2011.

20. D. Vekris, C. Dima. Efficient Operational Semantics for $\mathrm{EB}^{3}$ for Verification of Temporal Properties. In Proc. of FSEN, Springer, 2013, to appear. 